\title{
Impact of Artificial Intelligence in Travel, Tourism, and Hospitality
}

\author{
Jacques Bulchand-Gidumal
}

\section{Contents}

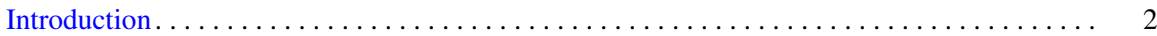

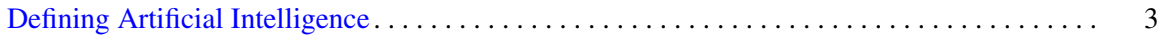

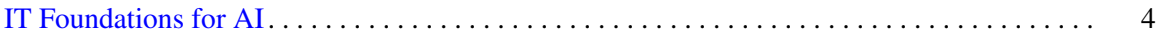

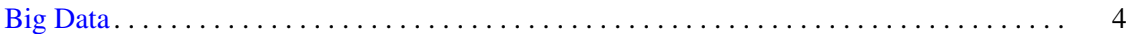

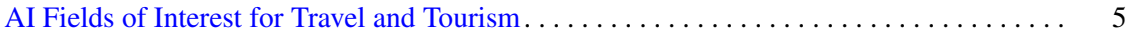

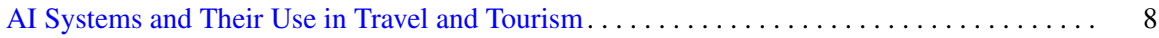

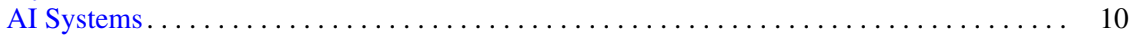

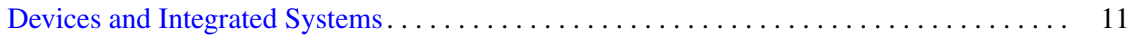

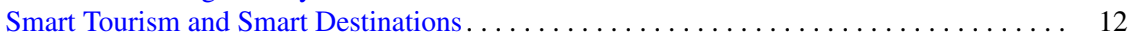

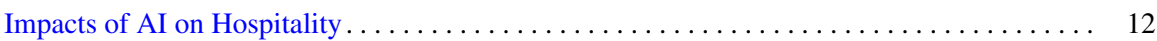

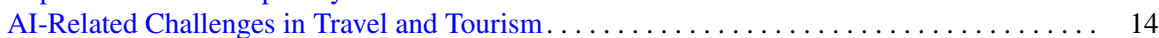

Issues Related to Tourists' Adoption and Use of AI . . . . . . . . . . . . . . . . . 14

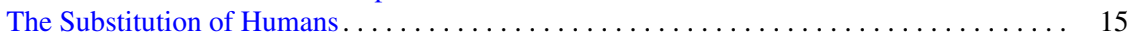

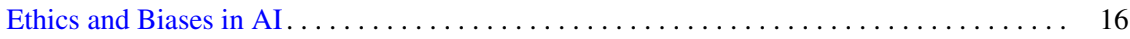

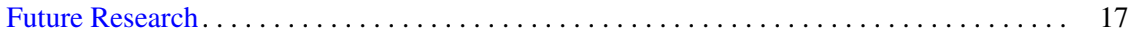

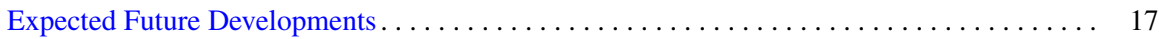

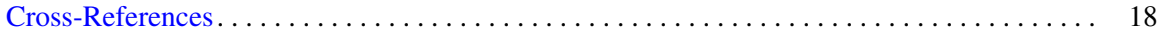

References.................................. 18

\section{Abstract}

Artificial intelligence (AI) is currently present in almost every area of travel and tourism, appearing in different types of applications such as personalization and recommender systems, robots, conversational systems, smart travel agents, prediction and forecasting systems, language translation applications, and voice

\footnotetext{
J. Bulchand-Gidumal $(\square)$

Institute for Sustainable Tourism and Economic Development (TIDES), University of Las Palmas de Gran Canaria, Las Palmas de Gran Canaria, Spain

e-mail: jacques.bulchand@ulpgc.es
} 
recognition and natural language processing systems. Recent improvements in big data, algorithms, and computing power have enabled significant enhancements in AI. In this chapter, we review how AI has changed and is changing the main processes in the tourism industry. We start with the IT foundations of AI that are relevant for travel and tourism and then address the AI systems and applications available in the sector. We then examine hospitality in detail, as a sector in which most of these systems are being implemented. We conclude with the challenges that AI faces in the tourism sector, a research agenda, and draw a scenario of the future of AI in tourism.

\section{Keywords}

Big data · Deep learning · Machine learning · Personalization · Forecasting · Robots

\section{Introduction}

Artificial intelligence (AI) relies on big data, processing capacities, and algorithms. Each of these three elements has experienced significant improvements lately, as several trends have coincided: first, the refinement of and advance in AI algorithms; second, significant improvements in processing capacities; and third, in the context of big data, the development of new and more powerful information sources and architectures that allow for the storing and processing of massive amounts of data. These improvements have, in turn, fueled significant enhancements in AI systems and robotics, in a process known as the Fourth Industrial Revolution (Li et al. 2019). Currently, AI applications are being developed and tested in all areas of the travel and tourism industry, including personalization and recommender systems, personal travel assistants, robots, prediction and forecasting systems, language translation applications, and voice recognition, and natural language processing systems.

Artificial intelligence is particularly relevant to travel and tourism for several reasons. Tourists need to make a series of decisions about future trips, for example, choosing a destination, transport, accommodation, and activities, among other things. These decisions will have a significant impact on tourists' satisfaction with their trip. However, the range of destinations, transport, accommodation, and activities currently available presents an almost infinite array of options necessitating assistance. Tourism organizations and agents face a similar challenge when trying to find the best match between customers and travel packages tailored to their needs. Organizations have an almost infinite supply of potential customers. Thus, matching demand with a product is an extremely complex process that seems well suited to the capabilities of AI. Once at their destination, tourists must navigate the realm of the unknown, characterized by differing habits, languages, cultural norms, and cuisine, among many other features that may be unfamiliar to them. Again, AI can help tourists in such "strange" environments, for example, recommending a 
travel itinerary or helping with language and cultural barriers. Also, AI can help organizations to personalize the experiences to tailor them to the desires of tourists.

While the tourism sector has been found to be an early adopter of most innovations, actual cases of AI use remain scarce. Most existing literature relates to laboratory scenarios and development cases. AI can currently be found embedded in data processing systems in real environments and at the production stage of various setups, for example, forecasting systems, robots, conversational systems, and voice recognition systems. However, AI is likely to become involved in all realms of the travel and tourism industry in the near future.

In this chapter, we review how AI has changed and is changing the main processes in the travel and tourism industry. We conceive of a future scenario where the current and future AI systems have been fully developed, deployed, integrated, and interconnected. We also examine the industry's challenges, especially privacy issues, workplace issues, and the deployment of the necessary connectivity.

\section{Defining Artificial Intelligence}

Before defining AI, we believe that it would be interesting to first clarify what intelligence means. Intelligence can be defined as a series of capabilities: the ability to understand the environment and the phenomena that occur, the ability to take advantage of past experiences, and the ability to combine the knowledge available to respond appropriately to a new challenge (Rudas and Fodor 2008). Gretzel (2011) summarizes these capabilities and says that intelligent systems are able to sense the environment, learn, and use what has been learned in future situations.

Artificial intelligence is usually defined as a set of technologies that can imitate human intelligence in the process of problem solving (Lai and Hung 2018). In the same vein that airplanes obtain the same result (flying) as birds but use entirely different mechanisms, AI tries to obtain similar results (thinking) as humans but through different mechanisms.

The concept of AI has evolved over time (Buhalis et al. 2019), from initial conceptualizations in which AI was defined as having some form of intelligence, to more recent definitions and conceptualizations in which AI is defined as being able to act autonomously on large amounts of data (Sterne 2017), to a future where AI could exceed human intelligence, an event that has been called the technological singularity (Kurzweil 2005). In this regard, the AI effect (McCorduck 2004) describes the phenomenon where as an AI application becomes mainstream, it stops being considered AI. This is because of the tendency to imagine that the application does not really contain AI (does not really think) but is just part of normal computing. Thus, the contents and limits of $\mathrm{AI}$ are dynamic over time.

Buhalis et al. (2019) refer to the four types of AI mentioned by Hintze (2016). The first type is reactive AI, which has no memory or use of the past. Deep Blue is the best example of reactive AI. The second type is limited memory AI, which has selective/limited recall. An example is how a self-driving car treats the objects around it. The third type is the theory of mind. These are machines that can represent 
other types of objects and their emotions, which would allow the machines to interact socially. The fourth type is AI with self-awareness or consciousness. Both Buhalis et al. (2019) and Hintze (2016) agree that the current challenges with AI are in the areas of extending artificial intelligence's memory, improving the ability to use past memories and experiences to make better decisions, and developing the capacity to process emotions and intuitions (Gretzel 2011). Transcending these four types of AI is the concept of superintelligence (Bostrom 2016). Superintelligence is defined as machine intelligence that surpasses general human intelligence.

Most current AI systems are domain specific. That is, they are systems capable of solving problems related to specific areas and specific tasks, such as spam filtering, understanding questions posed by humans, visually navigating a known environment, or even driving an autonomous car. This type of AI has been defined as "Weak AI" or "Narrow AI" (Russell and Norvig 2016). Future developments will create general purpose AI or "Strong AI" (Russell and Norvig 2016), that is, AI of the third and fourth types mentioned in the previous paragraph (systems that have intelligence in more than one area, with consciousness and the capacity to think). Recently, the concept of "Hybrid AI" (Wirth 2018) has emerged. Hybrid AI is situated between Strong AI and Weak AI, to include AI that exceeds Weak AI but without all the capacities of Strong AI. The travel and tourism sector requires Hybrid AI and Strong AI due to the vast array of tasks and elements that need to be integrated in order to develop the best possible experience for tourists.

\section{IT Foundations for AI}

Artificial intelligence systems require four basic elements to work: data, programs, hardware, and interconnectivity between the different systems. We will not analyze hardware in detail because the motto is that the more powerful hardware is, the better. Artificial intelligence applications usually require large hardware capacities (processing and storage) to run adequately, although there are certain hardware architectures that are more well suited for AI. Regarding interoperability, one of the key features of AI is its ability to have machines interoperate (Bowen and Morosan 2018; Gretzel et al. 2015), thus automating the aggregation and consolidation of data from multiple sources (Buhalis and Leung 2018).

We will now analyze in detail the cases of data and algorithms for AI.

\section{Big Data}

Big data form one of the key IT foundations of AI since big data provide the necessary input for AI systems to improve by learning, to find and understand patterns of behavior, and to generate insights. Big data can be defined as a series of datasets with a very large volume, generated at high speeds from a variety of sources and with multiple types of data (De Mauro et al. 2016). Big data is usually defined by four Vs: volume, velocity, variety, and veracity. Some definitions include 
two more Vs: value and volatility. These features are relevant and must be carefully considered, since the term big data is frequently used wrongly. Sometimes a process is tagged as big data simply because it uses a database with a large number of records. However, if there is only one source for all these records, or if they all represent the same type of transaction (e.g. hotel reviews in a social media website, transactions with an online travel agent), it would not be appropriate to call this process big data.

Big data in tourism usually comes from two sources: the environment and the tourist. The environment is the source of meteorological data, events occurring at the destination, and information obtained in real time from sensors, Internet of Things (IoT), and transactions. Tourists provide data before, during, and after their trip in five ways: online activities, offline activities, biometric and emotional data, wearables, and user-generated content (UGC). Before and during the trip, users search and book services online, and their digital footprint can be traced (Gunter and Önder 2016). During the trip, users also leave offline traces (e.g., movements, booking, consumption), which are captured by several devices: GPS data, mobile roaming, data from Bluetooth devices like beacons, IoT, and PoS. Also during the trip, biometric and emotional data from users can be automatically gathered (e.g., thermal images, face recognition). Likewise, and depending on user's granting access, data from wearables (e.g., smart watches, activity trackers, clothing) can be collected during the trip. Lastly, UGC is generated during and after the trip. Such content includes online reviews, comments in social networks, and pictures and videos posted online ( $\mathrm{Li}$ et al. 2018). User-generated content requires AI processing before it can be properly used by researchers and analysts. Artificial Intelligence can help in processing sentiment analysis in textual information (Schuckert et al. 2015) and analyzing and tagging characteristics of pictures, audios, or videos that are shared by users (e.g., place, participants, sentiments). These techniques greatly enhance UGC as a data source, by providing much richer information to the processes that use these data.

A user profile can be created by joining these data sources together. This profile can then be used to recommend products and services that are tailored to the user's needs. A summary of all the mentioned data sources and process of data generation for the specific case of hospitality can be found in Fig. 1.

\section{Al Fields of Interest for Travel and Tourism}

Artificial intelligence has a vast array of subfields, depending on the specific goals, tools, or methodologies that are used. We briefly explore the subfields that are of greater interest for travel and tourism.

\section{Ambient Intelligence}

Ambient intelligence (AmI) is "[...] about sensitive, adaptive electronic environments that respond to the actions of persons and objects and cater for their needs." (Aarts and Wichert 2009, 244). For example, a hotel room may adapt the 


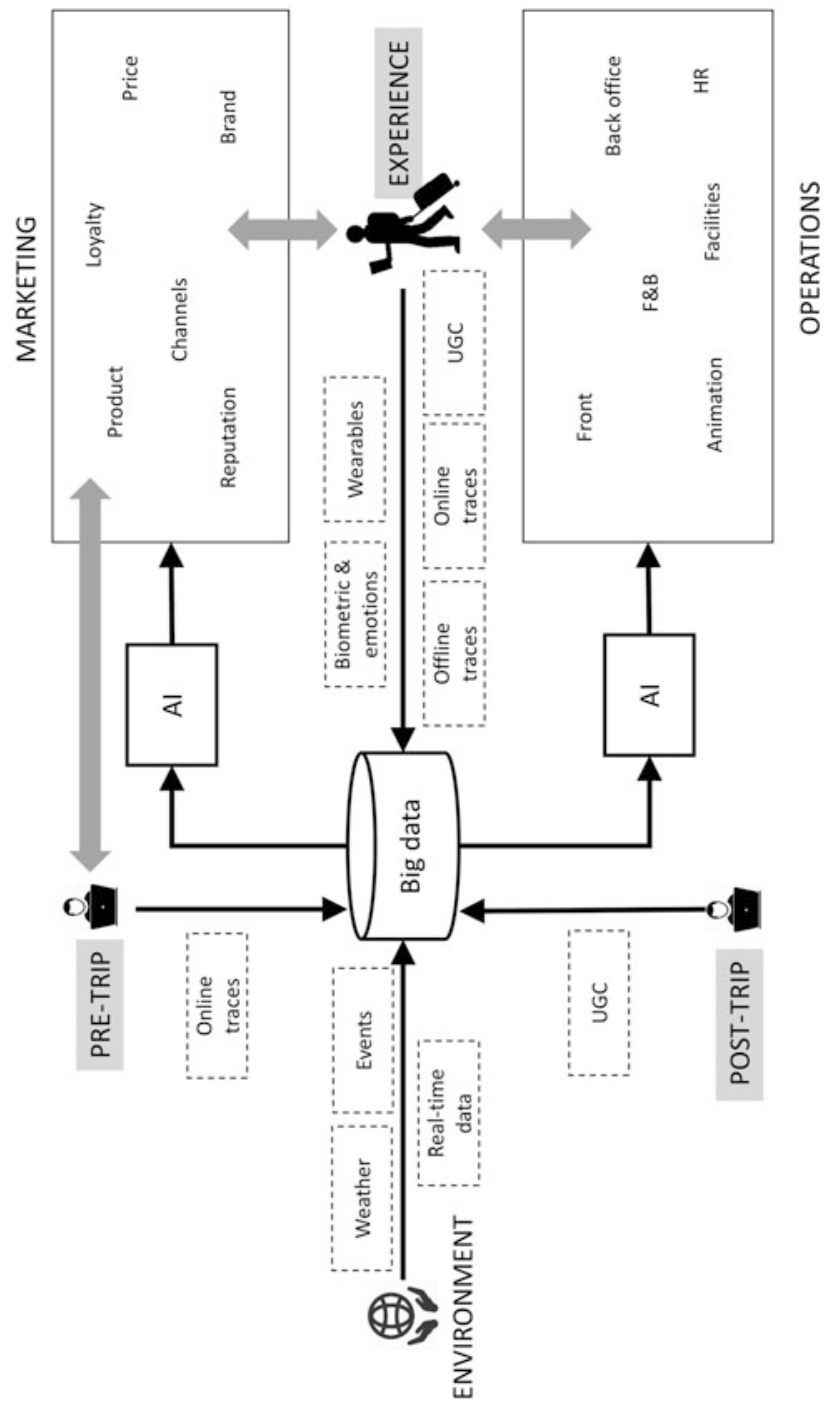

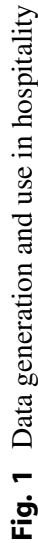


temperature, music, and light to the user's desire. It could even autonomously sense the need for these adaptations, without specific requirements from the user. The system can adapt the light in the room during the day, according to the user's activity. Ambient intelligence is not just suitable for private and small spaces such as hotel rooms but can also be used in large public spaces, such as an airport or a concert venue. Ambient intelligence can also be used to guide tourists (Basiri et al. 2018), based on crowdsourced data in which patterns are recognized.

\section{Natural Language Processing and Facial Recognition}

Natural language processing (NLP) allows computers to process natural language properly. The input can be through text or voice. In the latter case, there would first be a voice recognition process, before the language processing occurs. Natural language generation is usually part of NLP, as they allow IT systems to maintain a conversation with the user. Natural language processing is one of the requirements of automated translation. The importance of NLP in tourism is high, since it enables virtual travel assistants, conversational systems, and robots (Tussyadiah and Miller 2019).

Face recognition is usually used to identify a person in a digital image or video. For example, it could be used in the check-in process to automatically recognize a guest. However, face recognition is not only useful for recognizing a specific person. It can also be used to count the number of people in a certain area and even to detect emotions in the people who pass by a certain point (e.g., happiness of those leaving the breakfast buffet).

\section{Machine Learning, Deep Learning, and Neural Networks}

Machine learning and deep learning are both part of AI, deep learning being a specific type of machine learning. Machine learning is a set of algorithms through which the machines learn, as they repeat certain processes and obtain feedback on how they performed in those processes. This feedback can be provided by humans or developed by the machine after observing the results of previous processes (e.g., losing or winning a chess game). The training is usually conducted with very large data sets, thus allowing for the algorithms to improve quickly. For example, a machine may be taught to choose the best picture from a set of similar pictures of a travel memory. After observing whether the customer engages with that chosen picture or album, the machine can improve the selection process for future instances.

Deep learning is a technique of machine learning based on neural networks. Unlike machine learning, where the algorithm is provided with a large set of rules, in deep learning, the computer is given a model than can evaluate examples and a small set of instructions on how to modify the model to make it stronger and more accurate. Thus, the analysis starts at a superficial level but moves onto more complex and deep layers in successive approaches (Bulchand-Gidumal 2016).

There are many uses of machine and deep learning in tourism, which are usually integrated in other set of algorithms or applications: forecasting, translation, weather 
predictions, sentiment analysis, fraud prevention, and image and video recognition (Ma et al. 2018). However, deep learning is also a foundation for many of the fields of interest that we have mentioned, such as speech recognition and object detection in AmI (LeCun et al. 2015).

Neural networks are a group of techniques that can be used for machine and deep learning. Thus, neural networks are a form of deep and machine learning. While we previously stated that technology-based (artificial) systems do not have to mimic the way that nature performs certain activities, such as flying or reasoning, one line of work in AI has been to try to imitate human neurons and their connections, through artificial neural networks (ANN), or simply neural networks. Artificial neural networks are networks of a large amount of simple artificial neurons, each of which imitate a human neuron. They are connected similarly to the way that human neurons are connected. The theory of neural networks is that as the magnitude of connected neurons approaches that of humans (approximately $10^{11}$ ), artificial and natural systems can perform similarly. Currently, the main use of neural networks in tourism has been related to forecasting (Claveria et al. 2015).

\section{Al Systems and Their Use in Travel and Tourism}

Artificial intelligence systems have several applications in tourism. From the consumer perspective, AI helps users to find better and more relevant information, gives them greater mobility, improves their decision-making, and, ultimately, provides a better tourism experience (Gretzel 2011; Tussyadiah and Miller 2019). From the business perspective, AI can be used in almost every aspect of management (Buhalis et al. 2019), especially in promotion and productivity (Tussyadiah and Miller 2019). Artificial intelligence is also expected to encourage more sustainable travel (Tussyadiah and Miller 2019), by influencing customers to have a more social perspective.

Artificial intelligence systems in the tourism industry can be stand-alone systems or embedded in existing applications and systems. These systems include recommender systems, personalization systems and techniques, conversational systems (chatbots and voice assistants), forecasting tools, autonomous agents, language translation applications, and smart tourism destinations. Although we analyze each system separately, it must be stated that tourists will usually interact with technologies that integrate several of these systems. For example, a guest may interact with a robot that integrates a conversational system, and, depending on the requirements, a recommender system, a personalization technique, or an autonomous agent. The dialogue with the user may be based on a chatbot or voice assistant.

Smart destinations are the ecosystems created in destinations in which these advanced technologies are deployed together with other social and organizational features, as will be described later in this section.

Figure 2 illustrates the relationship between the IT foundations of AI analyzed in the previous section and the AI systems and applications that are examined here. 


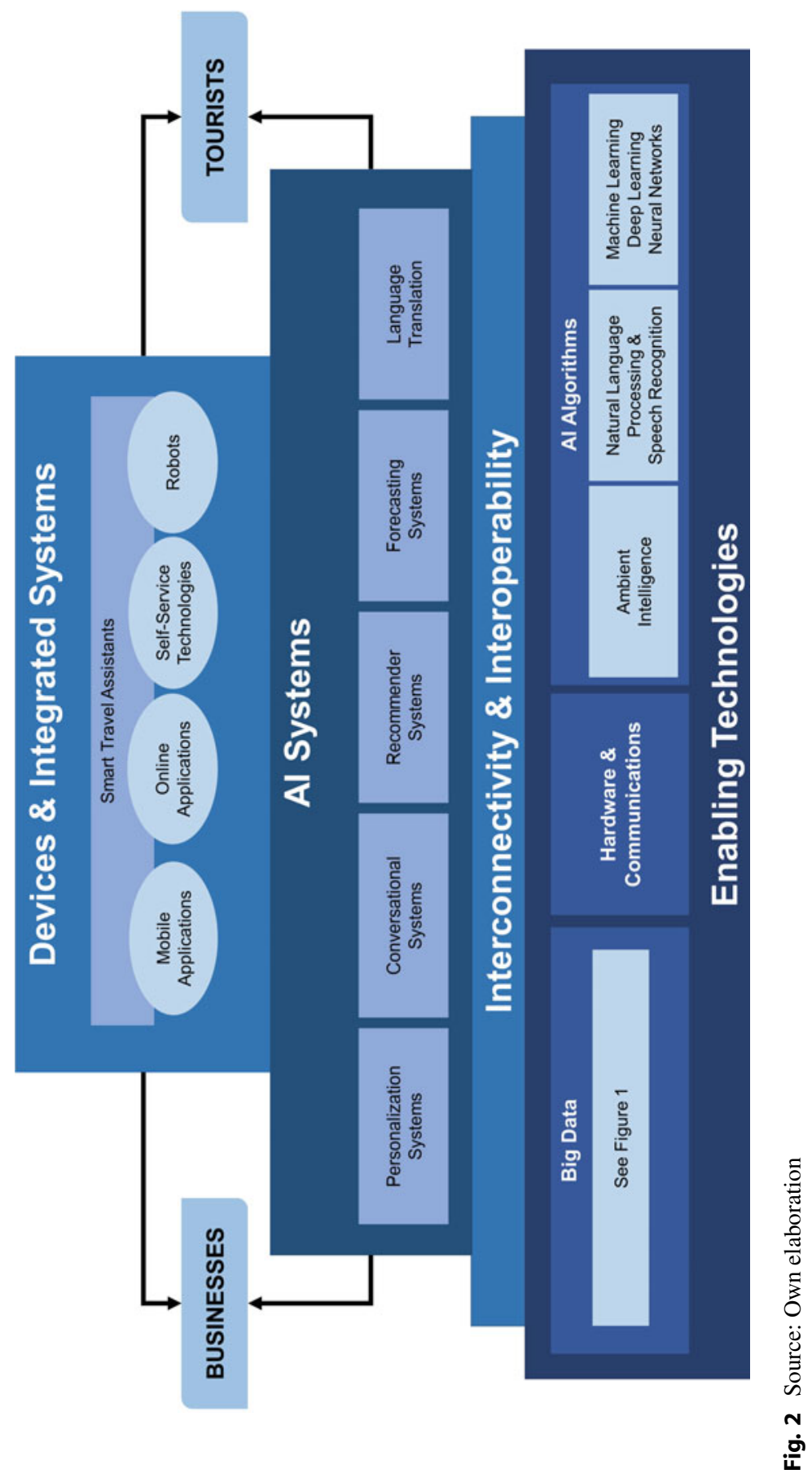




\section{Al Systems}

\section{Personalization and Recommender Systems}

For many years, tourists had to decide on the destinations to visit, places to visit in the destination, and the activities at the destination by using pictures in catalogues. The Internet has increased the quantity of information available, and UGC has also helped tourists to make better-informed decisions. However, even with this additional information, price has been the most important component in decision-making. Artificial intelligence changes this behavior, since it allows tourists to find the alternatives that best suits them and allows businesses to tailor their experiences to their customers' specific requirements. It does so through personalization techniques and recommender systems.

Recommender systems are tools and techniques oriented toward giving travelers options that best fit their interests (Ricci et al. 2015). The use of recommender systems in tourism has become increasingly important as the number of options available to users has grown exponentially with online environments (Gavalas et al. 2014). Usually, recommender systems match the characteristics of available options with user profiles in order to make suggestions about the most suitable options.

Personalization techniques try to provide users with customized information based on their preferences and restrictions (Gao et al. 2010). Thus, personalization techniques mean that companies change from marketing to many to marketing to one. Personalization techniques require large amounts of information about user behavior, so that an accurate profile can be defined. Gao et al. (2010) analyze in detail the theories, techniques, and applications for personalization.

\section{Conversational Systems: Chatbots and Voice Assistants}

Conversational systems allow customers to engage in a conversation which is usually related to information search. These conversations can span a long period of time and involve several processes (Gretzel 2011). Conversational systems are sometimes referred as chatbots or virtual agents (Buhalis et al. 2019). They involve technologies such as NLP and speech recognition and are currently ubiquitous. For example, they exist as personal assistants in smartphones and home speakers (with commercial systems such as Apple Siri, Google Assistant, Microsoft Cortana, and Amazon Alexa) and as textual chatbots in websites and kiosks. These systems are becoming the reference point, as less effort is required for users to communicate with them, and they present an experience closer to how humans naturally communicate. Melián-González et al. (2019) explain the determinants of chatbot usage by tourists.

\section{Forecasting}

Forecasting is a technique in which historical and contextual data is used to make estimates about the future, based on current trends. It is used in all types of sectors and business, in order to make decisions that require a prediction of what will happen. Forecasting is particularly well suited for AI algorithms (Claveria et al. 2015), especially with the presence of big data (Gunter and Önder 2016). Artificial 
intelligence methods in forecasting can be divided into five categories: grey theory, fuzzy time series, rough sets approach, support vector machines (SVMs), and ANNs (Claveria et al. 2015).

In the tourism sector, forecasting can be used to understand tourist demand (Buhalis and Leung 2018), to develop marketing strategies, for financial management and human resource allocation (Claveria et al. 2015; Huang 2014), to detect scams in restaurants (Stalidis et al. 2015), and to support the management of facilities and maintenance needs (Buhalis and Leung 2018).

However, the use of AI must be handled carefully, since the results of AI methods have been mixed. On the one hand, Yu and Schwartz (2006) found that complex models are not more accurate than simple, traditional models. Claveria and Torra (2014) had more promising results, although the quality of the forecasting results with neural networks was severely moderated by the degree of preprocessing. On the other hand, several studies have found that AI methods had better prediction accuracy. For example, Sun et al. (2019) used machine learning to forecast tourist arrivals, Law and Au (1999) used neural networks for a similar purpose, and Huang (2014) also used neural networks to forecast resort demand.

\section{Language Translation Applications}

Travel and tourism usually involve coming into contact with different languages. However, language has been found to be one of the main barriers that tourists face when travelling, as well as one of the sources of discomfort and anxiety (Cohen 2004). In many cases, language barriers also prevent tourists from exploring the local culture, as they adhere to franchises and known brands while abroad. As personalization can help tourists find new places (Benckendorff et al. 2019), automatic translation can facilitate the tourists' navigation of the destination, allowing them to explore and engage in all types of activities. Artificial intelligence that is empowered by machine learning and NLP is helping the development of automatic translation applications and simultaneous translation systems.

\section{Devices and Integrated Systems}

\section{Robots}

A robot is an autonomous machine (a physical object) that includes AI and senses the environment, both of which allow the robot to make decisions and perform actions. Physicality differentiates robots from other AI programs, and autonomy differentiates robots from ATMs, check-in kiosks, and other similar devices. Robots can be embodied in several forms (Tung and Law 2017): human-like, animal-like, object, or functional.

Traditionally, robots were found in industrial settings. However, AI has allowed robots to appear in service environments (Ivanov and Webster 2017), to the point that these service robots are able to overcome many shortcomings of humans in tourism, such as language barriers and labor shortages (Bowen and Morosan 2018). Ivanov and Webster (2017) mention two types of service robots: professional service 
robots and personal service robots. These AI-enabled professional service robots are being used to streamline processes and enhance tasks that have been traditionally performed by front office staff ( $\mathrm{Li}$ et al. 2019).

\section{Smart Travel Assistants}

As artificial intelligence, mobile devices, natural language processing, and speech recognition have improved, the concept of smart travel assistants has gained traction and feasibility. These assistants are applications that are familiar with the user (i.e., his/her preferences, interests, availability) and are thus able to provide suggestions on-demand or autonomously, anticipating the user's needs. These systems have also been called autonomous agents, intelligent travel agents, and smart concierges. An assistant should be able to combine several services at a destination, taking into account time and space restrictions, and find suitable ways to take the user from one place to the other within a desired budget.

One challenge regarding travel assistants is the question of the system's final owner. Currently, mobile apps and systems are usually used for free. In many cases, large corporations (e.g., Google, Facebook) pay for the system's costs. Therefore, the question is whether the travel assistant will be serving the tourist or the system's developer. In this regard, a new type of marketing is expected to be developed in the next few years, which could be called travel assistant marketing, that is, marketing oriented to travel assistants instead of to tourists.

\section{Smart Tourism and Smart Destinations}

Smart tourism can be defined as "[...] tourism supported by integrated efforts at a destination to collect and aggregate/harness data derived from physical infrastructure, social connections, government/organizational sources and human bodies/minds in combination with the use of advanced technologies to transform that data into on-site experiences and business value-propositions with a clear focus on efficiency, sustainability and experience enrichment." (Gretzel et al. 2015, 181). Artificial intelligence thus has a critical role in the development and deployment of smart tourism, since the transformation of data into experiences and value propositions will be empowered by AI.

That is, smart tourism and smart destinations are digital ecosystems in which AI play a key role. Nevertheless, in order to develop these ecosystems, there are several other social and organizational components that have to work and interact together.

\section{Impacts of Al on Hospitality}

Since hospitality is one of the main industries in tourism, we decided to analyze the impact of AI on hospitality in a more detailed manner, by examining the technologies and applications mentioned previously in the context of hotels. In order to review the AI applications that are currently being used, or under development, or 
will be implemented in a foreseeable future, we have divided the hospitality industry into two main areas: operations and marketing.

In the field of operations, AI allocates rooms and other resources according to guest value, helps in the preventive management of the facilities, bases the animation offer on the past behaviors and predictions of tourist arrivals, adapts the cuisine available to the tastes of the existing customers, enables room cleaning robots, helps select the appropriate employee for the facilities and the products offered, facilitates intelligent systems by enabling natural conversations with guests (e.g., at check-in, in-service demands), allows for the integration of dynamic information into business processes, facilitates the use of robots in the front desk, as concierges, and for delivery, improves stock management, improves energy management of the facilities and tourist consumption, enables the creation of an environment in which the guest can feel at home, provides guests with access to their own digital services, and supports finance management by taking into account expected revenues and arrivals.

In the field of marketing and commercialization, $\mathrm{AI}$ improves forecasting, adjusts prices and offers made to existing and potential customers, enhances customer relationship management (CRM) systems, helps develop personalized services and experiences through mass customization, allows the deployment of intelligent marketing, helps with the development of customized predictions, support agents and smart sales assistants, creates offers in real time that are sent to the user through a context-based and content-based approach, and allows for marketing to be used as a queue management tool.

One specific challenge that hotels must face is that big data is one of the foundations of the deployment of AI. However, it is difficult to describe the data sets available to hotels as big data. While the volume of data available to hotels is usually high, and there is some variety to the data, the data is usually limited to the guest's interaction with the hotel website before the trip, and his/her behavior in the hotel. Hotels have little data on their guests' profile, their interests and preferences, their preferred destinations and other characteristics, and their behavior outside the hotel. This means that hotels can only have big data about their guests by pairing with other businesses that can complement their data.

In the final stage, when AI is fully developed and implemented in the hospitality industry and the systems are all integrated and can interoperate, almost all the tasks that are currently performed by humans will be able to be performed by robots, AI, and natural language systems. However, this does not mean that the hospitality industry will be run without the presence of humans. Humans will continue to have two main functions. First, humans will develop a small set of tasks that are extremely difficult to automate, even with the development of the capabilities of robotics and AI. Second, the presence of humans will be used as a distinction and luxury; it will be a differential factor. In other words, if humans are inefficient from an economic perspective, their presence will be justified from a differentiation perspective or through an increase in quality, as is currently the case in gas stations.

Figure 3 illustrates the continuum that different hotels will be located on. On one end of the continuum is the efficient hotel that fully takes advantage of the 


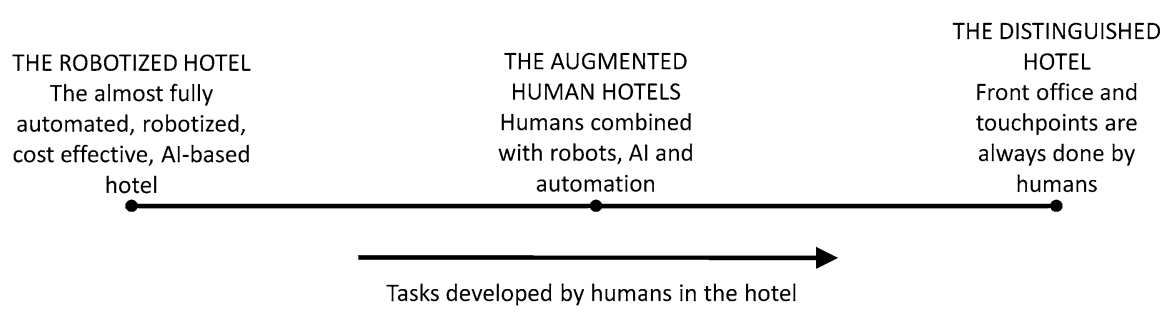

Fig. 3 AI technologies and application in tourism

capabilities of technology, automation, robots, and AI. It requires a limited amount of humans in its operation. These efficient hotels will be attractive to value conscious guests (Bowen and Morosan 2018), because they can save the main costs of the hospitality industry (Gursoy 2018). At the other end of the spectrum is the distinguished hotel, in which humans will be used in different touch points as a way of differentiation. However, even in these distinguished hotels, guests will always have the option of using self-service technologies based on AI, if they prefer to do so. In between, there will be several types of hotels in which different combinations of humans and technology will be found.

\section{Al-Related Challenges in Travel and Tourism}

Artificial intelligence currently has and will have many positive effects on the travel and tourism sector. However, there are some challenges and risks that have to be addressed. We analyze in detail three main issues: the tourists' perspective of AI, the substitution of humans by machines, and the ethics and biases in AI. We finish this section with some suggestions for future research.

\section{Issues Related to Tourists' Adoption and Use of Al}

The first challenge with AI is the tourists' thoughts, attitudes, and perceptions of these technologies. As with any other technology or innovation, tourists can be grouped into the categories mentioned by Rogers (2010): innovators, early adopters, early majority, late majority, and laggards. Taking into account the risks and benefits of AI, Tussyadiah and Miller (2019) found three types of users: laggards (who perceive high levels of risks and low levels of benefits for AI), aficionados (who perceive high levels of benefits and low levels of risks for AI), and realists (who are aware of both the likely benefits and risks of AI). These authors found that that the people with negative sentiments toward AI are those who have not used such technologies before (Tussyadiah and Miller 2019). Regarding user adoption of robots, as is the case with other technologies, the main drivers are perceived 
usefulness and performance (Bowen and Morosan 2018). However, Gretzel (2011) warns that there is a need to move from research on intentions of use, to research on actual use, use patterns, and nonuse.

Artificial intelligence has several benefits and risks for users. For tourists, one primary benefit of $\mathrm{AI}$ is that it can help them navigate unknown environments, thus reducing the anxiety and fear that tourists often feel (Buhalis et al. 2019). It could also help them develop new and memorable experiences (Li et al. 2019).

As for the risks, the three main concerns of tourists relate to the fear of surveillance, an AI divide and of a society entirely guided by technology. Regarding the fear of surveillance, several authors (Gretzel 2011; Tussyadiah and Miller 2019) have mentioned the threat to privacy that AI systems can pose, because they gather massive amounts of data, and, most importantly, have the ability to derive patterns and information from the data.

As happened with the digital divide at the beginning of the century caused by lack of access, there is a risk that there will be an AI divide. This AI divide could be caused by the reluctance of some users to participate in AI environments, due to their perceptions of risks in AI systems.

As for the concern regarding a society fully guided by technology, tourists will probably have to choose between more automated, efficient, and cost-efficient services and less automated and human-based luxury services. For example, hotels will probably be located on a continuum, as described above.

\section{The Substitution of Humans}

The substitution of the human workforce by machines has been taking place since the First Industrial Revolution. However, for a long time, machines were only able to replace humans in simple, routine tasks. With the growth of AI and AI-empowered technologies, a new generation of machines has appeared (such as service robots) that can now compete with and replace humans in almost every possible task (Brynjolfsson and McAfee 2011). This means that the tourism sector, which for a long time had been immune to this situation, is now at risk. Bowen and Morosan (2018) estimate that $25 \%$ of the workforce in hospitality could be replaced by robots in the next decade, thus categorizing the adoption of robots as a paradigm shift. Some traditional functions in tourism (such as the front desk of hotels) could even disappear (Bowen and Morosan 2018). Some authors have named AI as the largest threat to mankind (Musk 2014).

In fact, worker displacement has been one of the main concerns regarding the impact of AI in tourism (Tussyadiah and Miller 2019), not only because of the loss of jobs but also because the worker's loss of a sense of belonging (Li et al. 2019).

There has been an intense debate in the academic literature over which jobs are more susceptible of being performed by machines. Sigala (2018) states that machines are good at complex reasoning and at algorithm-based and repetitive tasks, while humans are best at generalization, perception, creativity, and interaction with 
the real world. However, as Sigala (2018) herself mentions, it is not the case that machines and technologies are unable to perform the tasks that humans are currently best at. It is simply that it is currently more expensive for computers to perform such tasks.

Therefore, although the initial consensus (based on industrial and office settings) was that low-skilled jobs were more threatened, the current data and research has a slightly different view (Brynjolfsson and McAfee 2011). The phenomenon of job polarization seems to point to a situation in which low-skilled and high-skilled jobs are the safest from automation, although the reasons are different for each cases. High-skilled jobs are safe because of the complexity of tasks and the existence of nonroutine tasks, while low-skilled jobs are safe because of the low costs of posts, the diversity of tasks, and the existence of nonroutine tasks. Instead, mediumskilled jobs, which are mostly associated with routine work, are more prone to being performed by technology (Melián-González et al. 2019).

Several authors (e.g. Brynjolfsson and McAfee 2011; Ivanov and Webster 2017) propose that in service contexts, technologies will not take over jobs but will instead be used to enhance employees and liberate them from routine tasks, thus allowing employees to have more time for better service. Artificial intelligence can thus augment their capacities, in a process that has been called AI augmentation and hybridization (Benckendorff et al. 2019).

In this regard, one of the main challenges of the tourism industry is that the tourism business could lose the sense of hospitality (Bowen and Morosan 2018), which is one of the main features of the tourism business.

\section{Ethics and Biases in Al}

The expected impact of AI on all aspects of life and society is massive. As part of the Fourth Industrial Revolution, its impact is comparable to that of machines and computers. This creates certain ethical challenges that need to be discussed. Two of these have already been mentioned, i.e., loss of privacy and fear of a society entirely guided by technology.

Another important and relevant risk associated with the widespread use of AI concerns bias. All humans are biased (including, obviously, those who create AI algorithms), and bias is natural in humans, e.g., bias relating to race, gender, age, and economic status. The problem is that AI is much more powerful than humans and might possibly facilitate amplification of the biases embedded in algorithms (Smith 2019). This could potentially mean that as AI learns, it learns to implement biased structures that are then replicated. In this sense, it has been recommended that AI systems should be transparent, robust enough to withstand manipulation and predictable (Bostrom and Yudkowsky 2014). Artificial intelligence systems will constantly have to make trade-offs, and they should be able to make balanced decisions that maximize the benefits for all participants. Lastly, if superintelligence (Bostrom 2016) systems are developed, the AI systems built should include ethics as a base feature (Bostrom and Yudkowsky 2014). 


\section{Future Research}

Apart from the questions mentioned in previous sections, AI development in the travel and tourism sector requires research in several areas. Three derive from similar challenges to those identified by Murphy et al. (2017) involving the use of robots in tourism and hospitality: firstly, customer acceptance of AI systems in travel and tourism; secondly, the impact of AI on the economics of the travel and tourism sector; and thirdly, the aforementioned effects of AI on the workplace and the substitution of humans. We have also identified another four questions: firstly, analysis of the ongoing impacts of AI on marketing and operations; secondly, changes in the working dynamics of the travel and tourism sector in areas such as product development and packet composition; thirdly, the impact that AI may have on sustainability (Tussyadiah and Miller 2019); and lastly, research into how information generated during travel and tourism activities can be integrated with data from other sources to better understand customer profiles and behavior.

\section{Expected Future Developments}

The future of AI in tourism is open. On the one hand, there is an optimistic view. In this view, society can address AI's main challenges. Privacy issues will be solved, connectivity will be implemented in order for AI systems to be deployed, and workers and AI systems will be able to work hand in hand. Under this paradigm, AI can be conceived of as a group of technologies that will enhance the tourism experience and make it better for all the actors. Businesses will be able to understand their customers better and thus design products, services, and experiences that are better tailored to their needs. It will also be possible for businesses to dynamically create personalized packages according to client interests. Technologies will replace and complement certain jobs, thus reducing overall operational costs and leading to savings that can then be partially passed on to customers. It also means that businesses will be able to offer services at an affordable price which might previously have been prohibitively expensive (Bowen and Morosan 2018). In other cases, technology can enhance particular jobs or free workers from certain tasks, improving service and customer support.

From the customer's perspective, AI will allow them to prepare their trips more quickly, with significantly lower transaction costs and a fully personalized package that suits their needs and interests. They will receive predictive offers that fit their requirements. During the trip, technologies will help tourists to navigate unknown environments seamlessly, reducing the anxiety and fear of the unknown. Language and cultural differences will not be barriers to tourism, but an additional attraction instead. Technologies will allow customers to receive the best possible service while guaranteeing privacy as much as possible (Bowen and Morosan 2018).

On the other hand, there is a less optimistic perspective. In this view, most of the industry's jobs will be substituted by machines, which will cause the loss of the 
hospitality feeling. There will be settings in which using machines is compulsory and not just an option, as is already occurring in many airports. Tourists will have to deal with machines and robots that are not ready to be used in a production environment and business will choose to use lower-cost products even if their performance is suboptimal. In most cases, technology will substitute humans, but the possible labor costs will not be translated to customers, who will pay the same getting a worse overall experience. The privacy and safety of data will not be guaranteed. Employees will find it difficult to work hand in hand with robots and AI systems, and organizations will not be entirely ready to adopt AI systems.

As with the cases of most paradigm-shifting technologies, it is likely that none of the two scenarios will be entirely accurate, and the future will be a combination of both.

\section{Cross-References}

$\checkmark \mathrm{AI}$ and the Travel Experience

- Artificial Intelligence and Machine Learning

$>$ Big Data

- Open and Commercial (Big) Data in Tourism

$\checkmark$ Post Smart Tourism

- Recommender Systems

- Robotics in Travel, Tourism and Hospitality

- Smart Destinations

- Smart Tourists and Intelligent Behavior

\section{References}

Aarts E, Wichert R (2009) Ambient intelligence. In: Bullinger HJ (ed) Technology guide. Springer, Berlin/Heidelberg, pp 244-249

Basiri A, Amirian P, Winstanley A, Moore T (2018) Making tourist guidance systems more intelligent, adaptive and personalised using crowd sourced movement data. J Ambient Intell Humaniz Comput 9(2):413-427

Benckendorff PJ, Xiang Z, Sheldon PJ (2019) Tourism information technology. CABI, Boston Bostrom N (2016) Superintelligence: paths, dangers, strategies. Oxford University Press, Oxford

Bostrom N, Yudkowsky E (2014) The ethics of artificial intelligence. In: The Cambridge handbook of artificial intelligence. Cambridge University Press, Cambridge, pp 316-334

Bowen J, Morosan C (2018) Beware hospitality industry: the robots are coming. Worldwide Hosp Tour Themes 10(6):726-733

Brynjolfsson E, McAfee A (2011) Race against the machine: how the digital revolution is accelerating innovation, driving productivity, and irreversibly transforming employment and the economy. Digital Frontier Press, Lexington

Buhalis D, Leung R (2018) Smart hospitality-interconnectivity and interoperability towards an ecosystem. Int J Hosp Manag 71:41-50

Buhalis D, Harwood T, Bogicevic V, Viglia G, Beldona S, Hofacker C (2019) Technological disruptions in services: lessons from tourism and hospitality. J Serv Manag 30:484-506 
Bulchand-Gidumal J (2016) Aprendizaje profundo y su impacto en turismo. In La actividad turística española en 2015:(edición 2016): 419-422. Síntesis

Claveria O, Torra S (2014) Forecasting tourism demand to Catalonia: neural networks vs. time series models. Econ Model 36:220-228

Claveria O, Monte E, Torra S (2015) A new forecasting approach for the hospitality industry. Int J Contemp Hosp Manag 27(7):1520-1538. https://doi.org/10.1108/IJCHM-06-2014-0286

Cohen E (2004) Contemporary tourism: diversity and change. Elsevier, Boston

De Mauro A, Greco M, Grimaldi M (2016) A formal definition of big data based on its essential features. Libr Rev 65(3):122-135

Gao M, Liu K, Wu Z (2010) Personalisation in web computing and informatics: theories, techniques, applications, and future research. Inf Syst Front 12(5):607-629

Gavalas D, Konstantopoulos C, Mastakas K, Pantziou G (2014) Mobile recommender systems in tourism. J Netw Comput Appl 39:319-333

Gretzel U (2011) Intelligent systems in tourism: a social science perspective. Ann Tour Res 38(3):757-779

Gretzel U, Sigala M, Xiang Z, Koo C (2015) Smart tourism: foundations and developments. Electron Mark 25(3): 179-188

Gunter U, Önder I (2016) Forecasting city arrivals with Google analytics. Ann Tour Res 61: $199-212$

Gursoy D (2018) Future of hospitality marketing and management research. Tour Manag Perspect $25: 185-188$

Hintze A (2016) Understanding the four types of AI, from reactive robots to self-aware beings. The conversation, Available at: http://theconversation.com/understanding-the-four-types-of-aifromreactive-robots-to-self-aware-beings-67616. Last accessed 17 July 2019

Huang HC (2014) A study on artificial intelligence forecasting of resort demand. J Theor Appl Inf Technol 70(2):265-272

Ivanov SH, Webster C (2017) Adoption of robots, artificial intelligence and service automation by travel, tourism and hospitality companies-a cost-benefit analysis. In: Artificial intelligence and service automation by travel, tourism and hospitality companies-a cost-benefit analysis. International scientific conference "contemporary tourism - traditions and innovations", Oct 2017, Sofia University, pp 19-21

Ivanov SH, Webster C, Berezina K (2017) Adoption of robots and service automation by tourism and hospitality companies. Revista Turismo Desenvolvimento 27(28):1501-1517

Kurzweil R (2005) The singularity is near: when humans transcend biology. Penguin, New York

Lai WC, Hung WH (2018) A framework of cloud and AI based intelligent hotel. In: Proceedings of the 18th international conference on electronic business, ICEB, Guilin, 2-6 Dec, pp 36-43

Law R, Au N (1999) A neural network model to forecast Japanese demand for travel to Hong Kong. Tour Manag 20(1):89-97

LeCun Y, Bengio Y, Hinton G (2015) Deep learning. Nature 521(7553):436

Li J, Xu L, Tang L, Wang S, Li L (2018) Big data in tourism research: a literature review. Tour Manag 68:301-323

Li JJ, Bonn MA, Ye BH (2019) Hotel employee's artificial intelligence and robotics awareness and its impact on turnover intention: the moderating roles of perceived organizational support and competitive psychological climate. Tour Manag 73:172-181

Ma Y, Xiang Z, Du Q, Fan W (2018) Effects of user-provided photos on hotel review helpfulness: an analytical approach with deep leaning. Int J Hosp Manag 71:120-131

McCorduck P (2004) Machines who think. A personal inquiry into the history and prospects of artificial intelligence, 2nd edn. A K Peters/CRC Press, Boca Raton

Melián-González S (2019) The impact of digital technology on work. Available at SSRN: https:// ssrn.com/abstract $=3353258$

Melián-Gonzalez S, Gutiérrez-Taño D, Bulchand-Gidumal J (2019) Predicting the intentions to use chatbots for travel and tourism. Curr Issues Tour. https://doi.org/10.1080/13683500.2019. 1706457 
Murphy J, Hofacker C, Gretzel U (2017) Dawning of the age of robots in hospitality and tourism: challenges for teaching and research. Eur J Tour Res 15:104-111

Musk E (2014) Available at https://twitter.com/elonmusk/status/495759307346952192. Last accessed 27 July 2019

Ricci F, Rokach L, Shapira B (2015) Recommender systems: introduction and challenges. In: Recommender systems handbook. Springer, Boston, pp 1-34

Rogers EM (2010) Diffusion of innovations. Simon and Schuster. New York

Rudas IJ, Fodor J (2008) Intelligent systems. Int J Comput Commun Control III(Suppl.):132-138

Russell SJ, Norvig P (2016) Artificial intelligence: a modern approach. Pearson Education Limited, Harlow

Schuckert M, Liu X, Law R (2015) Hospitality and tourism online reviews: recent trends and future directions. J Travel Tour Market 32(5):608-621

Sigala M (2018) New technologies in tourism: from multi-disciplinary to anti-disciplinary advances and trajectories. Tour Manag Perspect 25:151-155

Smith CS (2019) Dealing with bias in artificial intelligence. Available at https://www.nytimes.com/ 2019/11/19/technology/artificial-intelligence-bias.html. Last accessed 17 Dec 2019

Stalidis G, Karapistolis D, Vafeiadis A (2015) Marketing decision support using artificial intelligence and knowledge modeling: application to tourist destination management. In: Kavoura A, Sakas DP, Tomaras P (eds) Procedia—social and behavioral sciences 175:106-113. Elsevier, Madrid

Sterne J (2017) Artificial intelligence for marketing: practical applications. Wiley, Hoboken

Sun S, Wei Y, Tsui KL, Wang S (2019) Forecasting tourist arrivals with machine learning and internet search index. Tour Manag 70:1-10

Tung VWS, Law R (2017) The potential for tourism and hospitality experience research in humanrobot interactions. Int J Contemp Hosp Manag 29(10):2498-2513

Tussyadiah I, Miller G (2019) Perceived impacts of artificial intelligence and responses to positive behaviour change intervention. In: Information and communication technologies in tourism 2019. Springer, Cham, pp 359-370

Wirth N (2018) Hello marketing, what can artificial intelligence help you with? Int J Market Res 60(5):435-438

Yu G, Schwartz Z (2006) Forecasting short time-series tourism demand with artificial intelligence models. J Travel Res 45(2):194-203 